\title{
LA COMUNIDAD FITOPLANCTÓNICA EN EL EVENTO DE SURGENCIA FRENTE AL MAR CARIBE CENTRO COLOMBIANO
}

\section{PHYTOPLANKTON COMMUNITY IN THE UPWELLING EVENT OFF CENTRAL COLOMBIAN CARIBBEAN SEA}

\author{
Andrés Franco-Herrera ${ }^{1}$ \\ Eduardo Andrés Torres-Sierra ${ }^{2}$
}

\begin{abstract}
RESUMEN
Con el objetivo de evaluar la estructura de la comunidad fitoplanctónica bajo la condiciones de surgencia y no surgencia en la costa central del Caribe colombiano y establecer su condición frente a este tipo de eventos oceanográficos a escala regional, se adelantaron cuatro muestreos siguiendo el método Eureliano, entre los meses de julio a septiembre de 2006 en la columna de agua $(0-50 \mathrm{~m})$, dos en condiciones de surgencia y los restantes en no surgencia. Se evaluó la composición, la abundancia, la dominancia y la diversidad fitoplanctónica a partir de muestras obtenidas con botella Van Dorn y arrastres horizontales. Se encontró que el grupo dominante fueron las diatomeas, específicamente las especies de los géneros Skeletonema, Chaetoceros, Coscinodiscus y Thalassionema, con densidades superiores a $50 \mathrm{cel} / \mathrm{L}$. No se detectaron claras diferencias en
\end{abstract}

${ }^{1}$ Biólogo Marino, Doctor Oceanografía, Profesor Titular, Programa de Biología Marina, Facultad de Ciencias Naturales, Universidad de Bogotá Jorge Tadeo Lozano, Sede Santa Marta. Carrera 2 No. 11-68, Edificio Mundo Marino, El Rodadero, Santa Marta. PBX: +(57) 5 4229334. e-mail: andres.franco@utadeo.edu.co.

${ }^{2}$ Estudiante tesista, Programa de Biología Marina, Facultad de Ciencias Naturales, Universidad de Bogotá Jorge Tadeo Lozano, Sede Santa Marta. Carrera 2 No. 11-68, Edificio Mundo Marino, El Rodadero, Santa Marta. PBX: +(57) 5 4229334. e-mail: eduardotorres@fastmail.fm. la composición específica fitoplanctónica bajo las dos condiciones oceanográficas, pero sí mayores densidades de células en ausencia de surgencia. Ecológicamente, el fitoplancton se caracterizó por una alta riqueza de especies y una baja dominancia, lo que se reflejó en una alta diversidad. Desde esta perspectiva, el fitoplancton, en el sistema de surgencia analizado, se constituye en una comunidad análoga a aquellas que se encuentran en las grandes surgencias permanentes de borde occidental de los continentes.

Palabras claves: surgencia, fitoplancton, diversidad, diatomeas.

\section{SUMMARY}

To evaluate the structure of the phytoplankton community under upwelling and non-upwelling conditions in the Colombian central Caribbean coast and to establish its conditions, compared with similar oceanographic events on a regional scale, four samples, taken between July and September 2006, two in upwelling and two in nonupwelling condition, were carried out along the water column $(0-50 \mathrm{~m})$ following the Eurelian method. Water samples with the Van Dorn bottle and horizontal tows were taken to determine phytoplankton composition, density, dominance and diversity. It was found that diatoms were the dominant group, specifically species of the genera Skeletonema, Chaetoceros, Bacteriastrum and Thalassionema, showing densities higher than 50cel/L. 
No clear differences were detected within the specific phytoplankton composition under both oceanographic conditions, but a higher cell density was evident for the non-upwelling one. From the ecological point of view, the phytoplankton is characterized by a high species richness, a low dominance and consequently by a high biodiversity. Thus, the phytoplankton community associated to the upwelling system of central Colombian Caribbean, emerges as an analog community to that found in the huge western permanent upwelling of the western border.

Key words: upwelling, phytoplankton, diversity, diatoms.

\section{INTRODUCCIÓN}

Las surgencias son afloramientos de aguas subsuperficiales llevadas hasta la superficie y removidas desde el área de transporte por el flujo horizontal (Bula-Meyer, 1990). Estos eventos pueden ocurrir en cualquier parte del océano, pero los más destacados se manifiestan a lo largo de las costas occidentales de los continentes, como se aprecia frente a California, Perú, Chile, Islas Canarias y Sudáfrica, regiones que se caracterizan por presentar una elevada producción pesquera (Barnes \& Hughes, 1988; Lalli $\mathcal{E}$ Parsons, 1993). Esta alta producción secundaria está soportada por la comunidad fitoplanctónica, la cual se ve favorecida por la fertilización de nutrientes que han sido preformados en las capas profundas del océano y que por el evento de surgencia alcanzan la zona eufótica, generando un alto crecimiento celular y biomasa autotrófica. El fitoplancton, se constituye en la principal fuente de materia y energía de la red trófica marina pelágica, fortaleciendo principalmente las comunidades que dependen de este primer eslabón.

El departamento del Magdalena hace parte del sector central de la costa Caribe colombiana, presenta una franja costera amplia de $220 \mathrm{~km}$ con diferentes ecosistemas que son afectados por la surgencia costera que se da en la región (CORPAMAG, 2004). Ésta, se encuentra modulada principalmente por la acción de los vientos Alisios del noreste que producen campos de vientos paralelos o perpendiculares a la línea de costa favoreciendo estos eventos de afloramiento, siendo especialmente significativos entre Punta Gallinas (Guajira) y Puerto Colombia (Atlántico, Bula-Meyer, 1990; Andrade E Barton, 2005). Este evento provoca cambios en las variables fisicoquí- micas de la columna de agua, generándose estabilidades bajas o negativas, bajas temperaturas y salinidades altas. Desde el punto de vista químico, los niveles de nutrientes inorgánicos transportados verticalmente hacia la capa fótica son relativamente bajos, contrario a lo esperado, siendo esta la principal diferencia con las ya mencionadas surgencias de borde occidental. Al parecer, estaría dado a que las aguas de afloramiento tienen su origen en Aguas Intermedias del Atlántico Norte AIAN (Ramírez, 1983). Estas últimas, se caracterizan por presentar bajas concentraciones de compuestos inorgánicos, producto del patrón global de circulación termohalina del océano, cuyas aguas no han acumulado altas concentraciones de nutrientes producto de la remineralización de la materia orgánica en compuestos preformados (Libes, 1992). Durante un evento de surgencia es típico encontrar especies de crecimiento rápido (i.e. oportunistas), principalmente del microfitoplancton, como diatomeas de los géneros Skeletonema, Chaetoceros, Bacteriastrum, Thalassionema y Pseudonitzschia (Franco-Herrera, 2001; Tigreros, 2001). Es factible que especies de menor talla, como el pico- y nanofitoplancton, se puedan también ver incrementadas, sin embargo, por la dificultad de su identificación, especialmente en la resolución óptica, se pueden ver subestimadas.

El estudio de las comunidades marinas afectadas por el evento de surgencia frente a las costas del departamento del Magdalena en el Caribe colombiano es puntual y limitado a información secundaria. Las descripciones con que se cuentan, coinciden con la importancia del afloramiento sobre comunidades macroalgales pero no microalgales (Bula-Meyer, 1977, 1985; Díaz-Pulido E Garzón-Ferreira, 2002), principalmente durante la época seca de diciembre a abril donde se evidencia este evento oceanográfico en gran escala. No existe una valoración de los procesos sucesionales en la comunidad fitoplanctónica, los trabajos realizados en el departamento, se han centrado básicamente a la comparación de la biomasa fitoplanctónica entre los periodos secos y lluviosos (Franco-Herrera, 2001; Tigreros 2001, Barragán et al. 2003, Campos et al. 2004), sin describir su estructura específica durante el evento de surgencia.

El objetivo de esta investigación fue evaluar algunos atributos estructurales de la comunidad fitoplanctónica presente en el evento de surgencia costera que se da frente al departamento del Magdalena, Caribe colombiano, en el período julio a septiembre de 2006, 
enriqueciendo de esta forma los registros que se poseen del área y comparando la estructura fitoplanctónica con otros focos de surgencias a nivel mundial (Álvarez et al. 1976, Rodríguez \& Varela, 1987; Santander et al, 2003; Pizarro et al. 2005), para establecer el grado de similaridad que poseen.

\section{MATERIALES Y MÉTODOS}

Área de estudio. El departamento del Magdalena está ubicado en la zona centro del Caribe colombiano, comprende desde la desembocadura del río Magdalena hasta la desembocadura del río Piedras en el límite oriental del Parque Nacional Natural Tayrona (PNNT). Posee una gran variedad de ecosistemas marinos tanto de carácter estuarino, gracias a la Ciénaga Grande de Santa Marta (CGSM), como oceánicos, debido a las estribaciones de la Sierra Nevada de Santa Marta en el PNNT, que permiten la llegada de aguas oceánicas cerca de la costa (Ramírez, 1990).

El régimen climático regional está definido por la acción de los vientos Alisios y el movimiento de la Zona de Convergencia Intertropical (ZCIT), precisando en gran escala dos períodos climáticos, un seco de diciembre a abril y uno lluvioso de mayo a noviembre; en este último, se intercala, por lo general, una transición seca entre julio y agosto denominado "veranillo de San Juan" (Ramírez, 1990). La descarga continental es de los ríos Magdalena, Manzanares, Gaira y Piedra, principalmente y de la CGSM, produciendo fertilización en la capa de mezcla, con su mayor aporte al finalizar el período lluvioso (noviembre y diciembre).

Durante los meses de diciembre a abril y julio a agosto, se presentan las mayores velocidades de los vientos alisios en la región del departamento del Magdalena, superando los 4,0m/s (Franco-Herrera, 2005), con direcciones perpendiculares $\left(120^{\circ}\right)$, o paralelas $\left(26^{\circ}\right)$ a la línea de costa (Márquez, 1982). Estos campos de vientos, tanto de velocidad como de dirección, generan la suficiente tensión sobre la superficie del agua, permitiendo el desplazamiento mar afuera de las capas superficiales y el desarrollo de afloramientos de aguas profundas ya sea por acción de vientos continentales o por transporte de Ekman, si los vientos son perpendiculares o paralelos a la línea de costa, respectivamente. En cualquiera de las dos situaciones, las aguas de alforamiento corresponden a las AIAN de profundidades cercanas o mayores a 100 m (Bula-Meyer, 1990).

Diseño muestreal. Se siguió el método Eureliano para el estudio de la columna de agua, el cual consistió en evaluar en un punto fijo el comportamiento de las variables físicas, químicas y biológicas. Así, se realizaron cuatro muestreos en las coordenadas $11^{\circ} 12^{\prime} 30^{\prime \prime} \mathrm{N}$ - 74 ${ }^{\circ} 15^{\prime} 00^{\prime \prime} \mathrm{W}$, a $1 \mathrm{mn}$ al noroeste del Morro de Gaira, Caribe colombiano (Figura 1), entre los meses de julio a septiembre de 2006, específicamente en las fechas 26-07-2006, 15-08-2006, 01-08-2006 y 26-09-2006. Se escogió este lugar por encontrarse dentro de la zona de influencia de la surgencia, por presentar una profundidad mayor a $40 \mathrm{~m}$ y por estar sometido directamente a la acción de los vientos alisios. En cada uno de los muestreos se realizaron dos ciclos, uno en la mañana y otro hacia el medio día, considerados original y réplica, tomando muestras de agua con botella Van Dorn a 0, 10, $20,30,40$ y $50 \mathrm{~m}$ de profundidad, con el fin de abarcar toda la capa de mezcla, ya que la termoclina permanente durante el período de estudio se encuentra entre los 40 y $45 \mathrm{~m}$ de profundidad (Blanco, 1988) y la penetración máxima de luz o zona fótica en la región alcanza cerca de los 50m (Franco-Herrera, 2005). De esta forma, se obtuvieron un total de 48 muestras de fitoplancton. Para establecer si los campos de vientos locales podrían o no favorecer surgencias, se trabajó con información satelital procesada del espectro de luz infrarrojo y visible del GOES-West y GOES-East para las variables humedad relativa y campos de vientos desde 600 a 950mb (CIMSS, 2006). Las muestras de agua obtenidas para cada profundidad fueron almacenadas en recipientes plásticos de 3L de capacidad, fijadas con solución de lugol $(0,05 \% \mathrm{v}: \mathrm{v})$ y refrigeradas en neveras de icopor. Así mismo, se realizaron arrastres verticales desde 50m hasta la superficie con una red cónica simple de poro de malla de $25 \mu \mathrm{m}$, para complementar la composición fitoplanctónica presente. La identificación de células se realizó a nivel de género y especie, utilizando para ello un microscopio óptico de luz transmitida Nikon E200 con objetivos de 40x y 100x. Las guías empleadas fueron las de Cupp (1943), Vidal \& Carbonell (1977), Balech (1988), Vidal (1995), Tigreros (2001) y Botes (2003).

La información, se organizó en una matriz de composición-abundancia por muestreo, la cual permitió describir la composición específica y por grupos mayores: diato- 


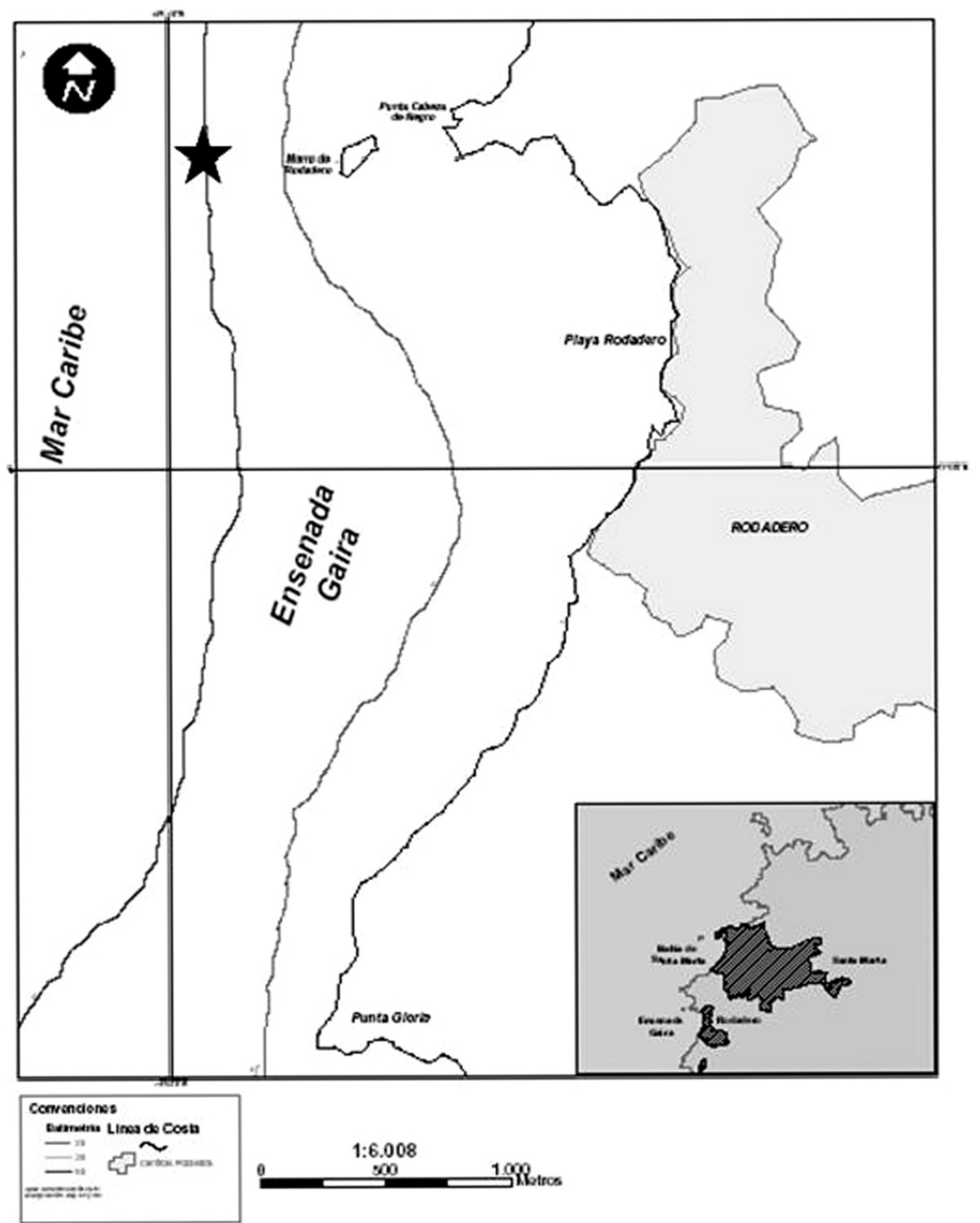

Figura 1. Ensenada de Gaira en el Caribe centro colombiano. La estrella indica las coordenadas del punto de muestreo donde se siguió el método Eureliano (Modificada de Franco-Herrera, 2005). 
meas, dinoflagelados y cianóbacterias. Igualmente, se calcularon los índices ecológicos de riqueza de especies, uniformidad de Pielou, diversidad de Shannon-Wiener y predominio de Simpson (Ramírez, 1999), con ayuda del paquete estadístico PRIMER 5 v.2,2 (Clarke, 1993).

Para detectar y describir la variación en la composición y la abundancia de la comunidad fitoplanctónica entre los muestreos, se realizaron análisis de clasificación cualitativos y cuantitativos, empleando los índices de similaridad de Jaccard y Bray-Curtis, respectivamente, usando como estrategia de ligamiento el promedio de grupos no ponderado (Clarke, 1993).

\section{RESULTADOS Y DISCUSION}

La información obtenida para los campos de vientos del satélite permitió establecer que dos muestreos se realizaron durante eventos de surgencia en las fechas 26-072006 y 15-08-2006 y los dos restantes en no surgencia para los días 01-08-2006 y 26-09-2006 (Arévalo et al. en rev.). Para el 26-07-2006, se presentaron patrones de velocidad superiores a $4,5 \mathrm{~m} / \mathrm{s}$ y dirección del viento NE-SO, que favorecieron el desarrollo de un evento de surgencia costera en la zona por transporte de Ekman, dado que los vientos presentan una direccionalidad paralela a línea de costa (Figura 2a). Durante el segundo muestreo el 01-08-2006, el viento mantuvo la misma dirección, pero la velocidad disminuyó marcadamente a niveles menores a $2,0 \mathrm{~m} / \mathrm{s}$, lo que generó una inhabilidad en el evento de afloramiento costero (Figura 2b). Para el 15-08-2006, la velocidad del viento se intensificó hasta niveles de $4,0 \mathrm{~m} / \mathrm{s}$ y la dirección fue perpendicular a la línea de costa, es decir, vientos del continente hacia el mar, provenientes de los cañones que se forman por las estribaciones de la Sierra Nevada de Santa Marta, los que favorecen eventos de surgencia por acción de vientos continentales (Figura 2c). Finalmente, en el cuarto muestreo, el día 26-09-2006, la presencia de vientos del E-NE con baja velocidad, impidieron el afloramiento de aguas profundas (Figura 2d). Esta tendencia de vientos es típica para el área de estudio, la cual se caracteriza por presentar vientos del noreste, correspondientes a los Alisios cuyas mayores intensidades se dan hacia los períodos secos (Márquez, 1982; Andrade, 1993). Estos campos de vientos son variables en el área de estudio, ya que la Sierra Nevada de Santa Marta causa flujos convergentes del aire del norte, noroeste y sureste con respecto a su eje principal (Kasakov et al. 1996), lo que hace que a nivel de la Ensenada de Gaira se puedan presentar vientos marinos paralelos o perpendiculares a la línea de costa que descienden hacia el mar en forma de un viento muy fuerte (brisa), pasando por el valle del río Piedras, el cerro de las Bóvedas, para llegar con especial intensidad hacia la Bahía de Santa Marta y la Ensenada de Gaira (Márquez, 1982).

Se identificaron un total de 86 especies fitoplanctónicas, correspondiendo el 53,4\% a diatomeas centrales, $24,1 \%$ a diatomeas pennadas y $19,76 \%$ a dinoflagelados. Las cianófitas y las clorófitas representaron menos del $2 \%$ de la composición específica. Las diatomeas Chaetoceros laciniosus, C. lorenzianus, Skeletonema costatum y Thalassionema nitzschoides fueron las especies más abundantes y frecuentes a lo largo del estudio, con densidades superiores a $2000 \mathrm{cel} / \mathrm{L}$ y frecuencias de aparición mayores al 65\%. Dentro de los dinoflagelados, los géneros Ceratium y Protoperidinium fueron los más representativos, pero sus densidades no superaron las $6 \mathrm{cel} / \mathrm{L}$. De igual forma, las cianófitas y las clórofitas representadas por los géneros Oscillatoria, Pediastrum y Scenedesmus, presentaron una frecuencia de aparición baja con densidades menores a 5cel/L. Esta misma tendencia de especies representativas, se mantuvo al integrar las abundancias fitoplanctónicas en la columna de agua, de tal forma que las densidades específicas fluctuaron entre 2,5 y $12962,4 \mathrm{cel} / \mathrm{m}^{2}$ (Tabla 1 ).

Durante los eventos de surgencia (S), las especies fitoplanctónicas más abundantes y frecuentes a lo largo de la columna de agua fueron Skeletonema costatum, Thalassionema nitzschoides, Chaetoceros laciniosus, C, lorenzianus, C. diversus, Coscinodiscus radiatus. Estas mismas especies, fueron significativamente más abundantes y frecuentes durante la ausencia de surgencia (NS), además de Asterionellopsis glaciallis, al punto que el $72 \%$ de la especies, se presentaron en los dos escenarios de muestreo (Tabla 1). Esto llevó a que el análisis de clasificación de presencia-ausencia de Jaccard mostrara una similaridad mayor al $60 \%$ en la mayoría de las muestras y a que se presentara una segregación parcial entre muestras obtenidas durante $S$ y NS (Figura 3a). 
a.

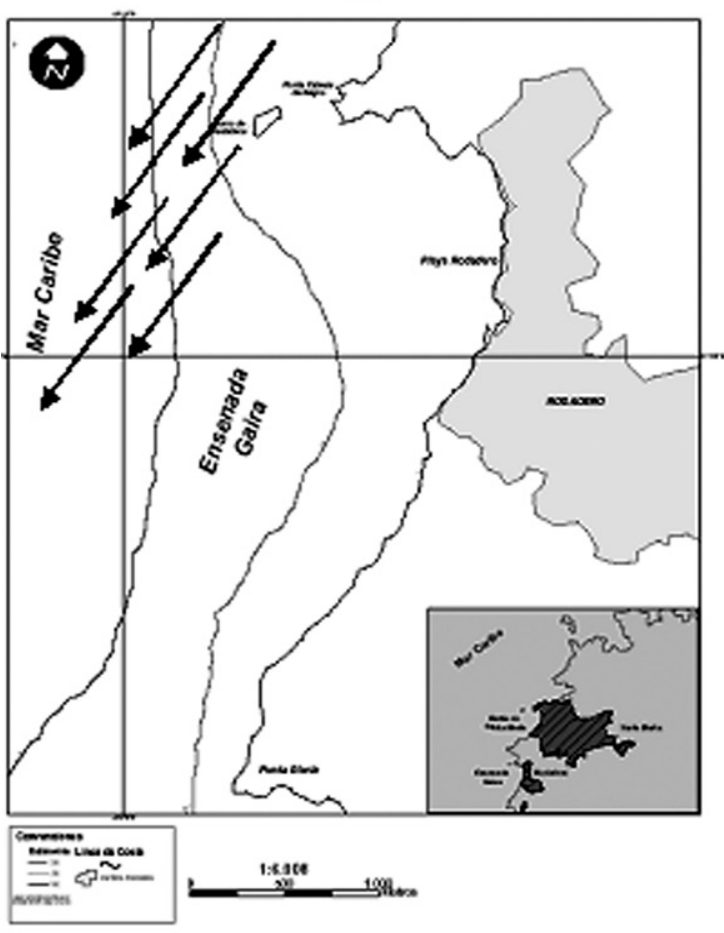

c.

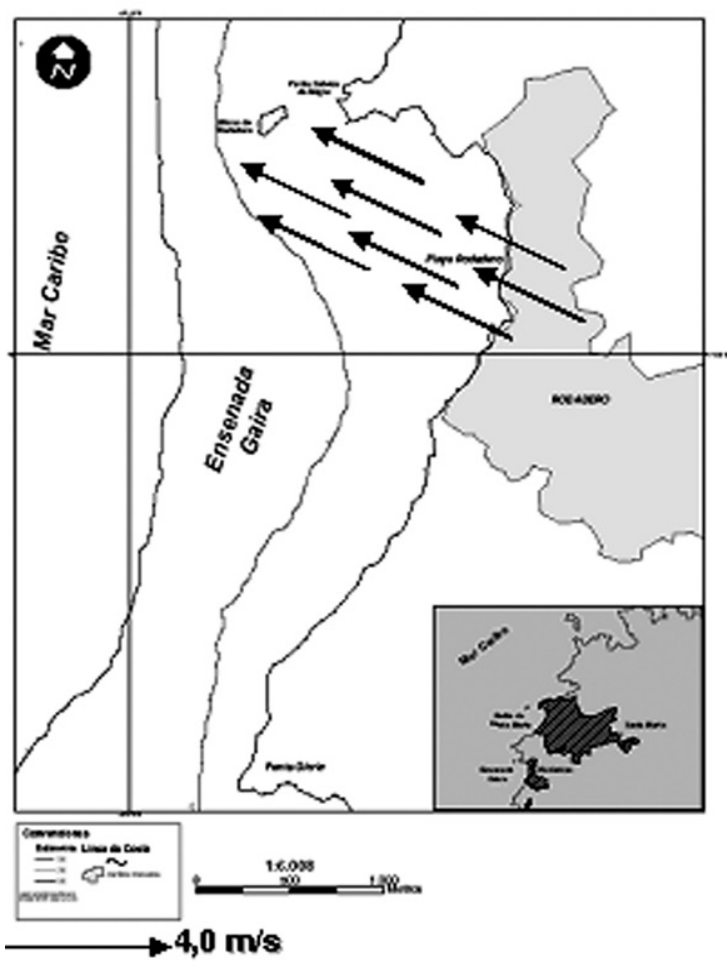

b.

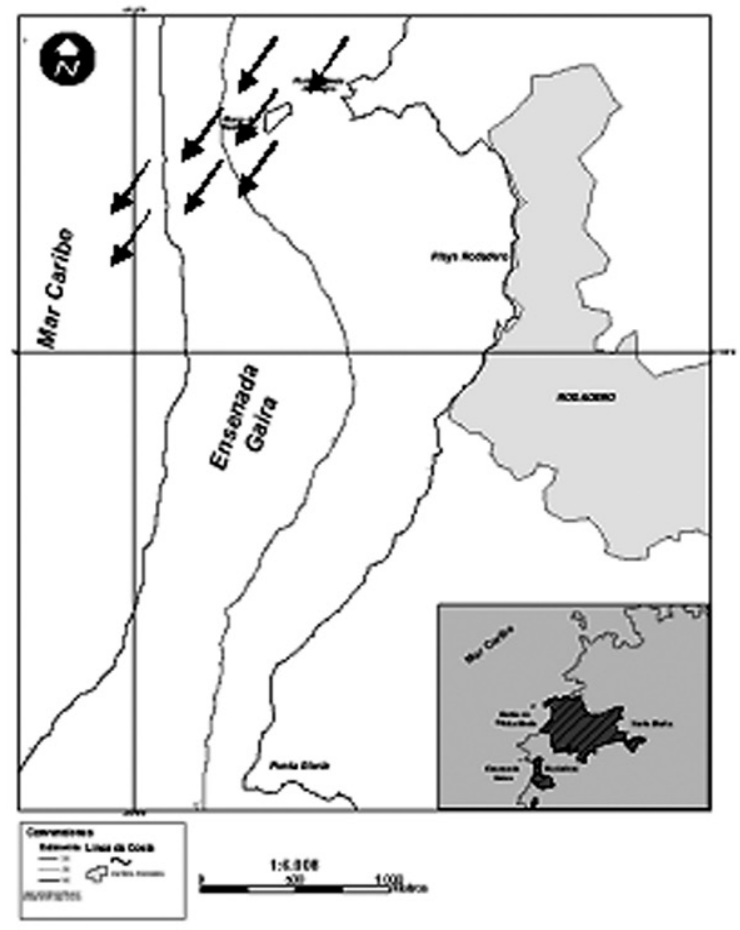

d.

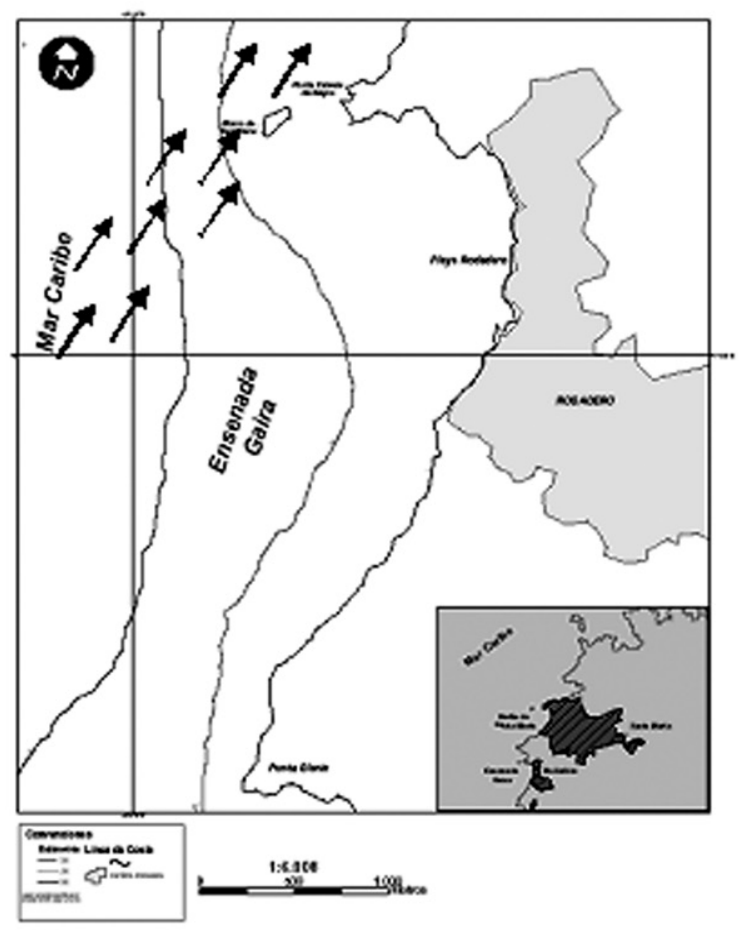

Figura 2. Campos de velocidad y dirección de vientos locales en la Ensenada de Gaira, Caribe colombiano, para los cuatro muestreos realizados entre los meses de julio y septiembre de 2006. a. 26-07-2006. b. 01-08-2006. c. 15-08-2006. d. 26-09-2006. Ensenada de Gaira en el Caribe centro colombiano. 
Tabla 1. Composición y densidad celular fitoplanctónica integrada para la columna de agua $\left(0-50 \mathrm{~m}, \mathrm{cel} / \mathrm{m}^{2}\right)$, para los muestreos realizados bajo condiciones de surgencia (S) y no surgencia (NS) en la plataforma continental en el Caribe centro colombiano. Números de 1-4, indican los ciclos totales realizados en cada condición oceanográfica.

\begin{tabular}{|c|c|c|c|c|c|c|c|c|}
\hline Especie & s1 & S2 & S3 & S4 & NS1 & NS2 & NS3 & NS4 \\
\hline Hemiaulus hauckii & 2,2 & 5,8 & 12,6 & 1,2 & 147,7 & 1,0 & 26,2 & 1,5 \\
\hline Hemiaulus sinensis & 2,1 & 7,6 & 15,2 & & 55,0 & 131,3 & 1,6 & \\
\hline Amphiprora gigantea & & & & & & 5,0 & & \\
\hline Amphiprora alata & & & 2,0 & 1,5 & 5,0 & 15,0 & & \\
\hline Bacteriastrum comosum & 4,4 & 43,4 & 137,5 & 118,4 & 737,8 & 791,3 & 76,7 & 4,0 \\
\hline Bacteriastrum delicatum & & & 91,6 & 185,7 & 162,7 & 255,0 & & 2,5 \\
\hline Chaetoceros affine & 112,7 & 78,7 & 656,7 & 457,2 & 3796,9 & 315,0 & 3,7 & 81,6 \\
\hline Chaetoceros curvisetum & 35,3 & 37,6 & 178,9 & 171,2 & 4746,0 & 2777,5 & 28,3 & \\
\hline Chaetoceros constrictus & 11,8 & 48,3 & 112,2 & 158,4 & 4181,6 & 1315,0 & 1,8 & 18,2 \\
\hline Chaetoceros diversum & 4,0 & 35,7 & 944,2 & 714,7 & 1511,3 & 1616,3 & 184,2 & 14,3 \\
\hline Chaetocero didymus & 15,2 & 3,0 & 39,6 & 222,9 & 3772,8 & 1932,5 & & \\
\hline Chaetoceros laciniosus & 657,7 & 2,6 & 2435,4 & 1997,7 & 12962,4 & 6961,3 & 246,3 & 159,2 \\
\hline Chaetoceros lauderi & & & 455,5 & 218,8 & 712,5 & 115,0 & 263,5 & 118,8 \\
\hline Chaetoceros leavis & & & 32,6 & 15,2 & 292,5 & 13,0 & & \\
\hline Chaetoceros lorenzianus & 21,9 & 24,4 & 2466,2 & 1526,4 & 7613,9 & 3632,5 & 33,7 & 356,8 \\
\hline Chaetoceros pelagicus & & 6,7 & & & & & & \\
\hline Chaetocero merranensis & & & 1,1 & 15,4 & 3,0 & 125,0 & & \\
\hline Chaetoceros peruvianum & 45,0 & 2,6 & 93,2 & 73,9 & 618,0 & 225,0 & 15,4 & 17,5 \\
\hline Chaetoceros sp. & 7,3 & 45,6 & 84,9 & 19,6 & 157,6 & 11,0 & 17,9 & 15,4 \\
\hline Corethron hyztrix & & & 18,0 & 1,3 & & & & \\
\hline Coscinodiscus gigas & 454,4 & 59,4 & 169,3 & 223,8 & 152,5 & 252,5 & 1,3 & 5,1 \\
\hline Coscinodiscus granii & 13,3 & 11,5 & 325,7 & 355,3 & 427,9 & 395,0 & 73,2 & 67,9 \\
\hline Coscinodiscus marginatus & & & & 1,3 & & & & 5,2 \\
\hline Coscinodiscus radiatus & 773,7 & 627,2 & 414,9 & 262,4 & 182,8 & 517,5 & 86,0 & 2,6 \\
\hline Stenophysis turris & & & 3,3 & & & & & \\
\hline Lithodesmus undulatum & & & 66,6 & 25,6 & 12,6 & 4,0 & 5,1 & 5,6 \\
\hline Odontella mobiliensis & 15,3 & 25,4 & 12,9 & 1,5 & 137,5 & 45,0 & & 5,2 \\
\hline Odontella sinensis & 15,1 & 22,5 & 17,8 & 1,1 & & & & \\
\hline Hemidiscus cuneiformis & 5,2 & 25,3 & 15,3 & 17,6 & 72,5 & 52,5 & & \\
\hline Leptocylindrus danycus & 8,4 & 35,2 & 284,4 & 19,9 & 24,4 & 122,5 & 1,2 & \\
\hline Guinardia flaccida & 5,2 & 25,8 & 87,0 & 1,1 & 212,6 & 12,5 & 72,0 & 39,1 \\
\hline Ditylium brightwelli & & & & 2,5 & 42,6 & & & \\
\hline Stenophysis turris & & & & & 2,0 & & & \\
\hline Rhizosolenia stolterfothii & 12,6 & 5,6 & 84,2 & 58,2 & 37,2 & 195,0 & 46,5 & \\
\hline Rhizosolenia alata & 12,5 & 2,6 & 57,5 & 65,9 & 18,2 & 1,0 & 33,6 & 51,7 \\
\hline Rhizosolenia faggilissima & & & & 3,2 & 15,0 & & & \\
\hline Rhizosolenia bergonii & 5,0 & 5,6 & & & & & & \\
\hline Rhizosolenia calcar-avis & & 5,0 & 5,0 & 3,5 & 4,4 & 15,0 & 17,9 & 1,3 \\
\hline Rhizosolenia castracanei & & 5,8 & & & & 5,0 & & \\
\hline Rhizosolenia hebetata & 5,0 & 2,5 & & & 3,0 & 5,0 & & 5,3 \\
\hline Rhizosolenia imbricata & & 25,4 & 144,0 & 4,9 & 137,7 & 35,0 & 23,5 & \\
\hline Rhizosolenia setigera & 15,4 & 5,8 & 137,3 & 99,0 & 182,5 & 132,5 & 5,1 & 2,9 \\
\hline Rhizosolenia robusta & 12,5 & 15,1 & 15,4 & 1,2 & & 1.0 & & 1,5 \\
\hline
\end{tabular}


Tabla 1. (Continuación)

\begin{tabular}{|c|c|c|c|c|c|c|c|c|}
\hline Especie & s1 & S2 & S3 & $\$ 4$ & NS1 & NS2 & NS3 & NS4 \\
\hline Thalassiothriz longissima & & & & 5,3 & & & & \\
\hline Skeletonema costatum & 41,9 & 21,8 & 6577,5 & 5453,3 & 7721,5 & 65,0 & 1977,7 & 1489,4 \\
\hline Asterionellopsis glacialis & 235,4 & 6,4 & 35,0 & & 27,0 & 895,0 & & \\
\hline Climacosphenia moniligera & 2,5 & & 15,0 & & 37,5 & 25,0 & & \\
\hline Corethron hyztrix & & & & 2,2 & & & & \\
\hline Grammatophora marina & & & 32,6 & 2,5 & 75,0 & & & \\
\hline Synedra spp. & 27,6 & 25,4 & 87,8 & 17,7 & 5,0 & 9,0 & 5,1 & 5,2 \\
\hline Thalassionema nitzschioides & 132,9 & 35,4 & 415,7 & 2994,4 & 4338,7 & 413,0 & 194,8 & 298,7 \\
\hline Diploneis crabro & 17,5 & 17,5 & 5,0 & & 15,0 & 27,5 & & \\
\hline Diploneis notabilis & & & 3,0 & & 25,0 & 1,0 & 5,1 & \\
\hline Gyrosigma spp & 35,1 & 15,3 & 56,5 & 25,4 & 52,5 & 147,5 & 5,1 & 5,2 \\
\hline Playotropic lepedoptera & & 5,8 & & & & & & \\
\hline Pleurosigma hamuleferum & & & & & 2,0 & 5,0 & & \\
\hline Pleurosigma elongatum & & & 61,6 & 22,6 & & 9,0 & 5,5 & \\
\hline Pleurosigma strigossum. & 12,7 & 17,7 & 144,3 & 43,4 & & 45,0 & 5,1 & 1,5 \\
\hline Pleurosigma nicobarium & & & & & 27,5 & & & \\
\hline Pleurosigma spp. & 1,0 & 17,7 & 122,9 & 2,2 & 11,0 & 22,0 & 21,9 & 1,2 \\
\hline Tropidoneis lepidoptera & & & 5,0 & 5,6 & & & & \\
\hline Trachyneis aspera & 1,4 & 22,8 & 2,0 & & & & & \\
\hline Nitzschia closterium & 22,6 & 1,1 & 6,0 & & 4,0 & 12,5 & 2,6 & 5,1 \\
\hline Nitzschia longissima & 5,0 & 5,0 & 5,0 & & & & & 5,1 \\
\hline Nitzschia pungens & 31,3 & 181,6 & 822,5 & 233,4 & 435,0 & 57,5 & 69,1 & 184,4 \\
\hline Nitzschia sigma & 25,8 & 15,2 & & & & 3,0 & 5,1 & 2,5 \\
\hline Ornithocerus magnificus & & & 5,6 & 5,1 & & & & \\
\hline Dinophysis caudata & 5,6 & 5,6 & & & & 2,0 & 5,5 & 5,2 \\
\hline Pyrocystis robusta & & & & & 15,0 & 25,0 & & 5,1 \\
\hline Ceratium lineatum & & 1,8 & & & & & & \\
\hline Ceratium carriense & & & 15,6 & & 5,0 & 5,0 & & \\
\hline Ceratium fusus & & 1,1 & 21,6 & 1,1 & & 5,0 & 5,1 & \\
\hline Ceratium inflatum & & & & & & & & 1,5 \\
\hline Ceratium massiliense & 1,0 & 5,8 & & 2,5 & 47,5 & 2,0 & 7,7 & \\
\hline Ceratium declinatum & & & & & & & 5,5 & \\
\hline Ceratium ranipes & & & & & & & & 5,3 \\
\hline Ceratium setaceum & & & 5,6 & & 4,0 & & & \\
\hline Ceratium tenue & & & & & & & 5,1 & \\
\hline Ceratium tripos & & 2,5 & 1,0 & 5,7 & 7,5 & 15,0 & & 5,2 \\
\hline Ceratium vultus & & & & & & & & 2,5 \\
\hline Protoperidinium conicum & & & & & & & 41,3 & 1,5 \\
\hline Protoperidinium divergens & & & & & & & 5,1 & 5,2 \\
\hline Protoperidinium oceanicum & 5,0 & 1,8 & 32,1 & & 7,5 & 32,5 & & 41,2 \\
\hline Pediastrum duplex & 22,7 & 25,4 & 5,6 & & 2,0 & 55,0 & & 16,0 \\
\hline Scenedesmus opoliensis & 65,0 & & 7,6 & 3,3 & & 1,0 & & \\
\hline
\end{tabular}



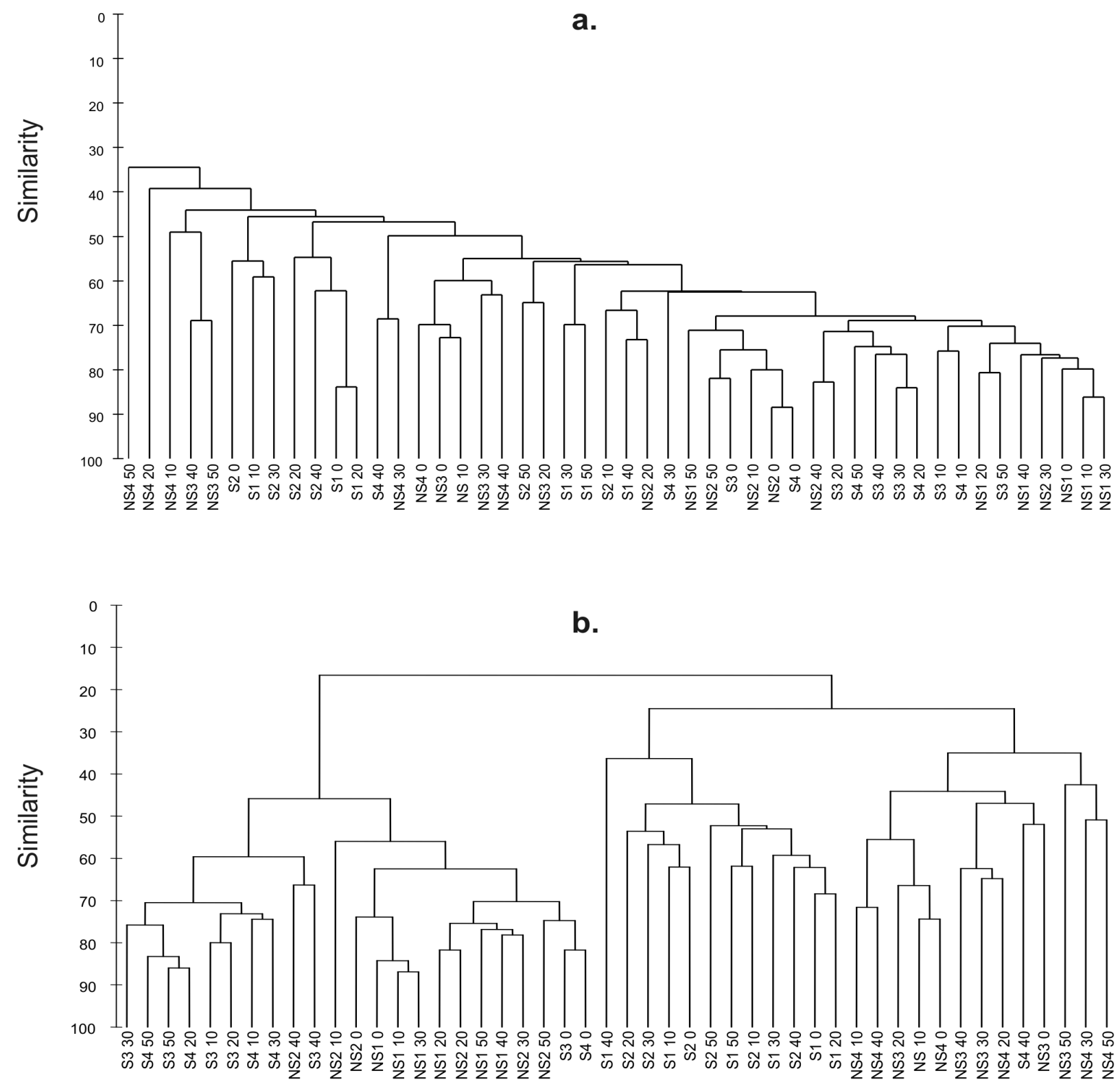

Figura 3. Análisis de clasificación cualitativo de Jaccard (a) y cuantitativo de Bray-Curtis (b) para muestras de fitoplancton obtenidas a diferentes profundidades en condiciones de surgencia (S) y no surgencia (NS) en la palataforma continental del Caribe centro colombiano. S ó NS + número + número = Surgencia o no surgencia + ciclo + profundidad.

Desde una perspectiva cuantitativa, la densidad promedio fitoplanctónica durante $\mathrm{S}$ fue de $234,25+/-$ 48,54cel/L, fluctuando en un rango de 36,96 a 987,50 cel/L. Concentraciones más altas, se obtuvieron durante NS, donde la densidad promedio alcanzó los 473,15 +/97,19cel/L, variando entre 26,38 y 1497,16cel/L. Igual comportamiento, se observó al integrar las densidades fitoplanctónicas en el estrato $0-50 \mathrm{~m}$, encontrándose que durante S las concentraciones estuvieron entre 2964,51 y $18110,05 \mathrm{cel} / \mathrm{m}^{2}$, mientras que en NS fueron superiores variando entre 3070,44 y 56007,49cel/ $\mathrm{m}^{2}$ (Tabla 2). Así, la principal diferencia entre los dos escenarios oceano- 
gráficos fue principalmente el cambio de densidad fitoplanctónica en la columna de agua, más que un cambio drástico en la composición específica, lo cual es reflejado en el análisis de similaridad cuantitativo de Bray-Curtis, donde se detecta con mayor claridad la segregación de muestras entre $S$ y NS, debido principalmente a los cambios de abundancia de las especies reportadas (Figura $3 \mathrm{~b}$ ). No obstante, las densidades encontradas son muy bajas comparadas con aquellas reportadas en otros sistemas de surgencia del Atlántico o Pacífico, donde la abundancia celular puede llegar a ser desde 100000 a 300000cel/L, como se presenta en los afloramientos de agua en la Bahía de Panamá (D'Croz et al. 1991) o alcanzar picos de hasta de 2'000.000cel/L, como sucede frente a las costas de Coruña en España (Casas et al. 1999). Incluso, para todo el Atlántico suroccidental, incluyendo áreas de surgencia, las densidades pueden variar entre 25000 a 200000 cel/L (Olguín et al. 2006). Similar panorama se presenta en el Pacífico tropical, donde las densidades fitoplanctónicas pueden fluctuar entre 100000 a 326000cel/L (Ramírez et al. 2006), demostrando aún más la baja densidad fitoplanctónica en el área de estudio. Sin embargo, las concentraciones son similares a aquellas presentes en diferentes regiones de la gran cuenca del Caribe, donde la abundancia celular puede fluctuar entre 180 a $1000 \mathrm{cel} / \mathrm{L}$, como sucede en la plataforma continental de Jamaica (Webber \& Roff, 1996) o desde 46 hasta $422 \mathrm{cel} / \mathrm{L}$ en el arco de las Antillas, en el Caribe oriental (Marshall, 1973).

Tabla 2. Atributos estructurales y ecológicos de la comunidad fitoplanctónica encontrada durante muestreos bajo condiciones de surgencia y no surgencia en la plataforma continental en el Caribe centro colombiano. $R=$ riqueza de especies, $\delta=$ uniformidad de Pielou, $\mathrm{H}^{-}=$Diversidad de Shannon-Wiener, $\lambda$ = Predominio de Simpson. M+número+letra = Muestreo+número+ciclo.

\begin{tabular}{|c|c|c|c|c|c|c|c|c|}
\hline & \multicolumn{4}{|c|}{ Surgencia } & \multicolumn{4}{|c|}{ No surgencia } \\
\hline & M1a & M1b & M2a & $\mathrm{M} 2 \mathrm{~b}$ & M1a & M1b & M2a & $\mathrm{M} 2 \mathrm{~b}$ \\
\hline $\mathrm{R}$ & 45 & 52 & 59 & 52 & 55 & 58 & 41 & 43 \\
\hline Densidad (cel/m²) & 2964,91 & 1682,87 & 18110,05 & 15873,03 & 56007,49 & 23908,00 & 3636,99 & 3070,44 \\
\hline$\delta$ & 0,75 & 0,74 & 0,61 & 0,60 & 0,65 & 0,65 & 0,57 & 0,59 \\
\hline $\mathrm{H}^{-}$ & 2,87 & 2,91 & 2,50 & 2,35 & 2,59 & 2,66 & 2,13 & 2,20 \\
\hline$\lambda$ & 0,09 & 0,10 & 0,15 & 0,17 & 0,11 & 0,10 & 0,27 & 0,24 \\
\hline $\begin{array}{c}\text { Géneros } \\
\text { representativos }\end{array}$ & \multicolumn{4}{|c|}{$\begin{array}{l}\text { Chaetoceros, } \\
\text { Coscinosdiscus, } \\
\text { Rhisozolenia, } \\
\text { Skeletonema, } \\
\text { Thalassionema }\end{array}$} & \multicolumn{4}{|c|}{$\begin{array}{l}\text { Chaetoceros, } \\
\text { Coscinosdiscus, } \\
\text { Rhisozolenia, } \\
\text { Skeletonema, } \\
\text { Thalassionema, } \\
\text { Asterionellopsis }\end{array}$} \\
\hline
\end{tabular}

La riqueza de especies encontrada es cercana a la reportada para otras regiones costeras del mundo donde se presentan eventos de surgencia. Santander et al. (2003), indican una riqueza de 87 especies en la zona costera de Iquique, entre Punta Cavancha y Bajo Molle en el Pacífico chileno, mientras que Ochoa E Tarazona (2003) identificaron 106 especies, de las cuales 68 fueron diatomeas y 38 dinoflagelados en la Bahía In- dependencia, Perú. De manera análoga, D’Croz et al. (1991) encuentran 55 especies en Bahía Panamá bajo condición de surgencia. Ahora bien, la dominancia de las diatomeas en número de especies y abundancias celulares, constituye la característica típica de una comunidad de ambientes de surgencia costera (Santander et al. 2003), ajustándose el Caribe centro colombiano a esta condición. Estudios previos ya había indicado esta 
tendencia, mostrando como especies dominantes a las diatomeas de los géneros Skeletonema, Chaetoceros, Bacteriastrum, Thalassionema y Pseudonitzschia, específicamente en el Golfo de Salamanca y el Parque Nacional Natural Tayrona (PNNT), zona influenciada por la surgencia costera del Caribe centro (Franco-Herrera, 2001; Tigreros, 2001). Incluso, es análogo a resultados obtenidos en otros focos de surgencia del Caribe colombiano, como lo es la Península de la Guajira, donde las abundancias relativas más altas corresponden a diatomeas de los géneros Bacteriastrum, Chaetoceros y Rhizosolenia (Duarte, 1996). Así mismo, en costas chilenas, se ha reportado como especie dominante a Thalassiosira subtilis, constituyente del orden centrales, mientras que en la plataforma peruana especies como Skeletonema costatum y Thalassionema nitzschioides son las dominantes (Santander et al. 2003; Ochoa E Tarazona, 2003).

La presencia de un alto número de especies de diatomeas de los géneros Chaetoceros o la abundante presencia de Skeletonema costatum o Thalassionema nitzschoides responde a que sus células, de un tamaño promedio de $20 \mu \mathrm{m}$, se encuentran embebidas en un mucílago, formando grandes agrupaciones. Hutchings et al. (1995) han señalado que las diatomeas que forman cadenas o colonias, con rangos de tamaño entre 5 y $30 \mu \mathrm{m}$, pueden tener una mayor habilidad para explotar escenarios de surgencia costera, ya que mantienen una alta eficiencia en términos de captación de luz, incorporación de nutrientes y crecimiento, dado que cada célula conserva su individualidad viéndose favorecida por una mayor relación superficie/volumen (Santander et al. 2003).

La diversidad calculada varió entre 2,13 y 2,91, sin encontrarse diferencias entre las comunidades fitoplanctónicas presente en S y NS. En ningún muestreo, se encontró el predominio de una especie o grupo de especies $(\lambda<0,27$, Tabla 2$)$, indicando así que la columna de agua alberga una comunidad altamente rica en especies, donde si bien algunos géneros presentan altas densidades, estas son contrarestadas por un alto número de especies con abundancias similares. Esto hace que la uniformidad $(\delta)$ encontrada sea superior al $56 \%$ en todos los casos (Tabla 2). Si bien los valores de diversidad pueden provenir de una composición específica variada, los rangos acá encontrados son similares a los reportados para comunidades fitoplanctónicas en focos de surgencia de la región norte y sur de Chile, donde se puede llegar a un valor de 2,5 (Santander et al. 2003; Pizarro et al. 2005). Tendencias similares se han encontrado en áreas del Pacífico colombiano, en cuyo foco de surgencia la diversidad puede llegar a los 2,5 ó 3,0 (Mora, 1993; Sánchez, 1996). Pinilla E Peña (2002) indican que el análisis de varias comunidades fitoplanctónicas de diferentes áreas oceánicas del Caribe, el Mediterráneo y el Atlántico Noroeste de África, han proporcionado una extensa serie de valores de diversidad, los cuales fluctúan entre 2,4 y 2,6 , es decir, que aquellos encontrados en esta investigación se ajustan en su mayoría a la condición regional de la cuenca del Caribe y Atlántico, incluso superando el rango máximo en muestreos bajo condiciones de surgencia.

En conclusión, la comunidad fitoplanctónica que se desarrolla en el ecosistema de surgencia durante el período agosto-septiembre de 2006 frente a las costas del departamento del Magdalena, se caracteriza por una alta riqueza de especies, principalmente de diatomeas centrales y pennadas, con una baja densidad fitoplanctónica y predominancia, así como una alta diversidad específica. La estructura comunitaria es similar a aquella encontrada en otros focos de surgencia alrededor del mundo en el borde occidental de los continentes. En ausencia de surgencia, el fitoplancton presente en la columna de agua del sector no difiere composicionalmente con aquel presente durante los afloramientos de agua, pero es evidente un incremento en la densidad celular, producto principalmente de la fertilización con nutrientes traídos por los aportes continentales. Se recomienda para futuros estudios en este tipo de ecosistema pelágico evaluar la sucesión fitoplanctónica cubriendo la época seca de diciembre a abril, cuando se da el evento de surgencia en una mayor escala temporal, realizando muestreos sistemáticos en la columna de agua cada hora, con el fin de tener una aproximación más detallada de la composición y la biodiversidad. Así mismo, estimar las concentraciones de carbohidratos y de proteínas fitoplanctónicas, para establecer su importancia alimenticia a las comunidades herbívoras del ecosistema pelágico, considerando así tanto la cantidad como la calidad nutricional de esta comunidad autotrófica. 


\section{AGRADECIMIENTOS}

El presente trabajo estuvo enmarcado dentro del Proyecto "Dinámica física, química y planctónica de la surgencia costera frente al Departamento del Magdalena, Caribe colombiano", del Programa de Biología Marina y la Dirección de Investigaciones de la Universidad de Bogotá Jorge Tadeo Lozano (UJTL), quien financió la totalidad del estudio (Código 315R). Los autores agradecen al personal de la UJTL por su apoyo en la ejecución de la investigación, a Adolfo Sanjuan Muñoz, M.Sc., por su ayuda en la revisión del documento y asesoría en campo y a los evaluadores anónimos, cuyos apreciaciones ayudaron a enriquecer y mejorar el manuscrito.

\section{BIBLIOGRAFÍA}

ÁlVAREZ, S.; LARA, J.R.; ACOSTA, M. 1976. Parámetros relacionados con la productividad orgánica primaria en dos antiestuarios de Baja California. California. Cooperative Oceanic Fisheries Investigations. Reports Volume XIX: 84-88.

ANDRADE, C.A. 1993. Análisis de la velocidad del viento en el mar Caribe. Bol. Cient. ClOH. 13:33-43.

ANDRADE, C.A.; BARTON, E.D. 2005. The Guajira upwelling system. Continental Shelf Research. 25 (9):1003-1022.

ARÉVALO, D.L., FRANCO-HERRERA, A. en rev. Características oceanográficas de la surgencia frente a la Ensenada de Gaira, Departamento del Magdalena. Sometido a: Boletín de Investigaciones Marinas y Costeras.

BALECH, E. 1988. Los dinoflagelados del Atlántico sudoccidental. Publicaciones especiales. Instituto Español de Oceanografía. Ministerio de Agricultura, Pesca y Alimentación. Madrid. 310p.

BARNES, R.S.K.; HUGHES, R.N. 1988. An introduction to marine ecology. Balckwell Science. Oxford. 351p.

BARRAGÁN, R.G.; MALDONADO, F.; NIETO, S.; FRANCO-HERRERA, A. 2003. Relación de la biomasa fitoplanctónica con la dinámica fisicoquímica y meteorológica de la capa de mezcla en la Bahía de Gaira, Caribe colombiano, durante el período Agosto-Noviembre de 2.003. Informe final. Sem. Invest. Fac. Biología Marina. U. de Bogotá Jorge Tadeo Lozano. Santa Marta. 57p.

BLANCO, J.A. 1988. Las variaciones ambientales estacionales en las aguas costeras y su importancia para la pesca de la región de Santa Marta, Caribe colombiano. Tesis de Maestría. Depto. Biología. Fac. de Ciencias. U. N. de Colombia. Santa Marta. 62p.

BOTES, L. 2003. Phytoplankton Identification Catalogue. Saldanha Bay, South Africa, April 2001. GloBallast Monograph. Series No. 7. IMO London. 77p.

BULA-MEYER, G. 1977. Algas marinas bénticas indicadoras de un área afectada por aguas de surgencia frente a la costa Caribe colombiana. An. Inst. Inv. Mar. Punta de Betín. 9:45-71.

BULA-MEYER, G. 1985. Un núcleo nuevo de surgencia en el Caribe colombiano detectado en correlación con las macroalgas. Bol. Ecotrópica. 14:3-20.

BULA-MEYER, G. 1990. Oceanografía: 100-113. En: FEN- COLOMBIA. Caribe Colombia. Bogotá. 264p.

CAMPOS, E.M.; OREJARENA, J.; PALOMINO, M.; FRANCO-HERRERA, A. 2004. Dinámica de la biomasa fitoplanctónica en la capa de mezcla y su relación con la oceanometeorología de la Bahía de Gaira, Caribe colombiano. Fase II: Febrero - Mayo de 2004. Informe final. Sem. Invest. Fac. Biología Marina. U. de Bogotá Jorge Tadeo Lozano. Santa Marta. 53p.

CASAS, B.; VARELA, M.; BODE, A. 1999. Seasonal succession of phytoplankton species on the coast of $A$ Coruña (Galicia, northwest Spain). Bol. Inst. Esp. Oceanogr. 15(1-4):413-429.

CIMSS (COOPERATIVE INSTITUTE FOR METEOROLOGICAL SATELLITE STUDIES). 2006. Tropical cyclones information. U. Wisconsin-Madison. Disponible desde Internet en: http://cimss.ssec. wisc.edu/tropic/. (con acceso: 26/07/06, 01/08/06, 15/08/06, 26/09/06). 
CORPAMAG (CORPORACIÓN AUTÓNOMA REGIONAL DEL MAGDALENA). 2004. Informe general del Departamento del Magdalena. Disponible desde Internet en: www.corpamag.gov.co/departamento. htm (con acceso 10/0504).

CLARKE, K. 1993. Non-parametric multivariate analyses of changes in community structure. Aust. J. Ecol. (18): 117-143.

CUPP, E. 1943. Marine plankton diatoms of the west coast of North America. Bull. Scripps Inst. Oceanography. U. California. La Jolla, California. 238p.

D'CROZ, L.; DEL ROSARIO, J.; GOMEZ, J. 1991. Upwelling and phytoplankton in the Bay of Panama. Rev. Biol. Trop. 39(2):233-241.

DÍAZ-PULIDO, G.; GARZÓN-FERREIRA, J. 2002. Seasonality in algae assemblages on upwelling-influenced coral reefs in the Colombian Caribbean. Botánica Marina. 45:284-292.

DUARTE, G. 1996. Estructura del fitoplancton al noroeste de la Guajira, durante el crucero Caribe 1993. Tesis de Grado. Fac. Biología Marina. U. de Bogotá Jorge Tadeo Lozano. Santa Marta. 98p. + anexos.

FRANCO-HERRERA, A. 2001. Estrategias de ingestión y flujo de carbono entre fitoplancton y Eucalanus spp. (Copepoda: Calanoidea) en dos ambientes oceanográficos del Caribe central colombiano. Centro de Investigaciones Científicas. Fac. Biología Marina. U. de Bogotá Jorge Tadeo Lozano. Santa Marta. 67p.

FRANCO-HERRERA, A. 2005. El Rodadero, más allá que un centro turístico. Una aproximación a la oceanografía de la Ensenada de Gaira. Servigraphics Ltda. Bogotá. 58p.

HUTCHINGS, L., PITCHER, G.; PROBYN, T.; BAILEY, G. 1995. The chemical and biological consequences of coastal upwelling. En: Santander, E.; Herrera, l.; Merino, C. 2003. Fluctuación diaria del fitoplancton en la capa superficial del océano durante la primavera de 1997 en el norte de Chile (20¹8’S):
II. Composición específica y abundancia celular. Rev. Biol. Mar. Oceanografía. 38(1):13-25.

KASAKOV, A.L., LEZHENIN, A.A., SPERANSKIY, L.S. 1996. Resultados preliminares del estudio de la capa límite mesometeorológica de la atmósfera en la costa norte colombiana aplicando un modelo numérico. Bol. Cient. CIOH. 17:17-26.

LALLI, C.M.; PARSONS, T.R. 1993. Biological oceanography. An introduction. Pergamon Press. Oxford. 301p.

LIBES, S.M. 1992. An introduction to marine biogeochemistry. John Wiley and Sons, Inc. Toronto. 734p.

MÁRQUEZ, G. 1982. Los sistemas ecológicos marinos del sector adyacente a Santa Marta, Caribe colombiano I: Generalidades. Ecol. Trop. 2(1):5-18.

MARSHALL, H.G. 1973. Phytoplankton Observations in the Eastern Caribbean Sea. Hydrobiologia. 41(1):45-55.

MORA, M.E. 1993. Contribución al conocimiento de los dinoflagelados de las zonas de surgencia oceánica y costera, durante condiciones anómalas de temperatura en el Pacífico colombiano (marzo-abril, 1992). Tesis de Grado. Fac. Biología Marina. U. de Bogotá Jorge Tadeo Lozano. Cartagena. 41p.

OCHOA, N.; TARAZONA, J. 2003. Variabilidad temporal de pequeña escala en el fitoplancton de Bahía Independencia, Pisco, Perú. Rev. Peru. Biol. 10(1):59-66.

OLGUIIN, H.F.; BOLTOVSKOY, D.; LANGE, C.B.; BRANDINI, F. 2006. Distribution of spring phytoplankton (mainly diatoms) in the upper $50 \mathrm{~m}$ of the Southwestern Atlantic Ocean (30-61 S). J. Plank. Res. 28(12):1107-1128

PINILLA, G.; PEÑA, V. 2002. Composición, distribución y abundancia de la comunidad fitoplanctónica de la ensenada de Utría, Pacífico colombiano. Revi. Biol. Mar. y Oceanografía. 37(1):67-81.

PIZARRO, G.; MONTECINO, V.; GUZMÁN, L.; MUÑOZ, V.; CHACÓN, V.; PACHECO, H.; FRANGÓPULOS, 
M.; RETAMAL, L.; ALARCÓN, C. 2005. Patrones locales recurrentes del fitoplancton en fiordos y canales australes $\left(43^{\circ}-56^{\circ} \mathrm{S}\right)$ en primavera y verano. Cienc. Tecnol. Mar. 28(2):63-83.

RAMÍREZ, D.G.; GIRALDO, A.; TOVAR, J. 2006. Producción primaria, biomasa y composición taxonómica del fitoplancton costero y oceánico en el Pacífico colombiano (septiembre-octubre 2004). Invest. Mar. 34(2):211-216.

RAMÍREZ, G. 1983. Características fisicoquímicas de la Bahía de Santa Marta (Agosto, 1980- Julio, 1981). An. Inst. Inv. Mar. Punta de Betín. 13:111-121.

RAMÍREZ, G. 1990. Distribución de los nutrientes inorgánicos en las aguas costeras de la región de Santa Marta, Caribe colombiano. Memorias VII Seminario Nacional de Ciencias y Tecnologías del Mar. Comisión Colombiana de Oceanografía: p.244-254.

RAMÍREZ, A. 1999. Ecología aplicada. Diseño y análisis estadísticos. U. Jorge Tadeo lozano. Centro editorial Escuela Colombiana de Ingeniería. Bogotá D.C. $325 p$.

RODRÍGUEZ, C.; VARELA, R. 1987. Pigmentos clorofílicos e hidrografía en la región noroccidental de Venezuela con énfasis en el Golfo de Venezuela. An. Inst. Inv. Mar. Punta de Betín. 17:3-14.

SÁNCHEZ, E. 1996. Caracterización espacial del fitoplancton nerítico en el Pacífico colombiano du- rante julio-agosto de 1994. Tesis de grado Fac. Ciencias. Universidad del Valle. Cali. 60p.

SANTANDER, E.; HERRERA, L.; MERINO, C. 2003. Fluctuación diaria del fitoplancton en la capa superficial del océano durante la primavera de 1997 en el norte de Chile (20 $18^{\prime}$ S): II. Composición específica y abundancia celular. Rev. Biol. Mar. y Oceanografía. Chile. 38(1):13-25.

TIGREROS, P.C. 2001.Biodiversidad y bioquímica del fitoplancton marino asociado a ambientes mesotróficos y oligotróficos del Golfo de Salamanca, Caribe colombiano. Tesis de grado. Fac. Biología Marina. U. de Bogotá Jorge Tadeo Lozano. Santa Marta. 173p. + anexos.

VIDAL, L.A.; CARBONELL, M.C. 1977. Diatomeas y dinoflagelados de la Bahía de Cartagena. Tesis de grado. Fac. Biología Marina. U. de Bogotá Jorge Tadeo Lozano. Bogotá. 360p.

VIDAL, L.A. 1995. Estudio del fitoplancton en el sistema lagunar estuarino tropical Ciénaga Grande de Santa Marta, Colombia, durante el año 1987. Tesis de Maestría. Depto. Biología. Fac. Ciencias. U. N. de Colombia. Santa Marta. 207p.

WEBBER, D.F.; ROFF, J.C. 1996. Influence of Kingston Harbor on the phytoplankton community of the nearshore hellshire coast, southeast Jamaica. Bull. Mar. Sc. 59(2):245-258.

Recibido: Febrero 28 de 2007. Aceptado: Mayo 15 de 2007 